\title{
Predictive analysis of photodynamic treatment depth with different photosensitizer based nanocarriers
}

\section{Análisis predictivo de la profundidad del tratamiento fotodinámico con nanoportadoras de diferentes fotosensibilizadores}

\author{
F. Fanjul-Vélez, I. Salas-García, J. L. Arce-Diego ${ }^{S}$ \\ Grupo de Técnicas Ópticas Aplicadas. Departamento TEISA, \\ Universidad de Cantabria, Av. de los Castros S/N, 39005 Santander, Cantabria, España. \\ (*) E-mail: fanjulf@unican.es $\quad$ S: SEDOPTICA member \\ Received: 11/09/2015 Accepted: 04/05/2016 \\ DOI: $10.7149 / 0 P A .49 .2 .48537$
}

\begin{abstract}
:
The effect of gold nanoparticles to deliver different types of photosensitizer used in Photodynamic Therapy on the depth of light penetration is studied by means of a predictive model with dosimetric purposes. The model employed allows to analyze the changes induced by the spherical nanoparticles administration in the optical distribution within the malignant tissue. The depth of photodynamic treatment was estimated in a nodular basal cell carcinoma taking into account both the conventional photosensitizer delivery and by means of nanoparticles. Significant differences were observed and demonstrate the great functionality of predictive modeling to rule out the use of certain nanoparticles to deliver the photosensitizer due to the limited therapeutic effect at certain depths within the carcinoma despite the photosensitizer delivery improvements.
\end{abstract}

Key words: Nanoparticles, photosensitizer, carcinoma, Photodynamic Therapy, optical propagation.

\section{RESUMEN:}

En este trabajo se estudia el efecto de las nanopartículas de oro sobre la distribución de la radiación óptica con fines dosimétricos, cuando actúan como portadoras de diferentes tipos de fotosensibilizadores usados en Terapia Fotodinámica. Para ello se emplea un modelo predictivo que permite analizar los cambios inducidos por la administración de nanopartículas esféricas sobre la distribución de la radiación óptica en el tejido maligno. A partir de la distribución óptica se estima la profundidad del tratamiento fotodinámico en un carcinoma basocelular nodular considerando tanto el fotosensibilizador convencional como el entregado mediante nanopartículas. Los resultados muestran diferencias significativas que demuestran la gran utilidad del modelado predictivo para descartar el uso de ciertas nanopartículas como portadoras del fotosensibilizador, ya que a pesar de favorecer una mejor acumulación del agente fotoactivo, limitan a priori el efecto terapéutico sobre carcinomas de cierta profundidad.

Palabras clave: Nanopartículas, fotosensibilizador, carcinoma, Terapia Fotodinámica, propagación óptica.

\section{REFERENCIAS Y ENLACES}

[1] D. K. Chatterjee, L. S. Fong, Y. Zhang, "Nanoparticles in photodynamic therapy: An emerging paradigm," Adv. Drug Deliv. Rev 60, 1627-1637 (2008). http://dx.doi.org/10.1016/j.addr.2008.08.003

[2] F. Scarmato, R. Fonseca, J. A. Thomazine, A. C. Tedesco, N. Lange, M. V. Lopes, "In Vitro Metabolism of 5-ALA Esters Derivatives in Hairless Mice Skin Homogenate and in Vivo PpIX Accumulation Studies," 
Pharmaceut. Res. 21, 2247-2252 (2004).

http://dx.doi.org/10.1007/s11095-004-7677-3

[3] H. S. Gill, S. N. Andrews, S. K. Sakthivel, A. Fedanov, I. R. Williams, D. A. Garber, F. H. Priddy, S. Yellin, M. B. Feinberg, S. I. Staprans, M. R. Prausnitz, "Selective removal of stratum corneum by microdermabrasion to increase skin permeability," Eur. J Pharm. Sci. 38, 95-103 (2009). http://dx.doi.org/10.1016/j.ejps.2009.06.004

[4] R. R. Allison, H. C. Mota, V. S. Bagnato, C. H. Sibata, "Bio-nanotechnology and photodynamic therapyState of the art review," Photodiagn. Photodyn. 5, 19-28 (2008). http://dx.doi.org/10.1016/j.pdpdt.2008.02.001

[5] M. R. Hamblin, P. Mróz, Advances in Photodynamic Therapy: Basic, Translational and Clinical. Engineering in medicine \& Biology (2008).

[6] M. E. Wieder, D. C. Hone, M. J. Cook, M. M. Handsley, J. Gavrilovic, D. A. Russell, "Intracellular photodynamic therapy with photosensitizer-nanoparticle conjugates: cancer therapy using a 'Trojan horse," Photochem. Photobiol. Sci. 5, 727-734 (2006). http://dx.doi.org/10.1039/B602830F

[7] M. K. K. Oo, X. Yang, H. Du, H. Wang, "5-aminolevulinic acid-conjugated gold nanoparticles for photodynamic therapy of cancer," Nanomedicine 3, 777-786 (2008). http://dx.doi.org/10.2217/17435889.3.6.777

[8] T. Vo-Dinh, Biomedical Photonics Handbook. Boca Raton: CRC Press (2003). http://dx.doi.org/10.1201/9780203008997

[9] S. L. Jacques, Monte Carlo Simulations of Fluorescence in Turbid Media, Handbook of Biomedical Fluorescence. B. W. Rogue and M. A. Mycek eds. CRC Press (2003).

[10] X. Xu, A. Meade, Y. Bayazitoglu, "Numerical investigation of nanoparticle-assisted laser-induced interstitial thermotherapy toward tumor and cancer treatments," Lasers Med Sci. 26, 213-222 (2011). http://dx.doi.org/10.1007/s10103-010-0828-3

[11] C. F. Bohren, D. R. Huffman, Absorption and Scattering of Light by Small Particles. John Wiley (1983).

[12] C. Mätzler, MATLAB Functions for Mie Scattering and Absorption. Universitas Bernensis (2002).

[13] E. Salomatina, B. Jiang, J. Novak, A. N. Yaroslavsky, "Optical properties of normal and cancerous human skin in the visible and near-infrared spectral range," J. Biomed. Opt. 11, 0640261-640269 (2006). http://dx.doi.org/10.1117/1.2398928

[14] B. C. Wison, M. S. Patterson, "The physics, biophysics and technology of photodynamic therapy," Phys. Med. Biol. 53, R61-R109 (2008). http://dx.doi.org/10.1088/0031-9155/53/9/R01

[15] I. Salas-García, F. Fanjul-Vélez, N. Ortega-Quijano, A. Lavín-Castanedo, P. Mingo-Ortega, M. LópezEscobar, J. L. Arce-Diego, "Effect of gold nanoparticles in the local heating of skin tumors induced by phototherapy," Proc. SPIE 8092, 8092041-7 (2011). http://dx.doi.org/10.1117/12.889830

[16] I. Salas-García, F. Fanjul-Vélez, J. L. Arce-Diego, "Influence of the human skin tumor type in Photodynamic Therapy analysed by a predictive model," Int. J. Photoenergy 2012, 1-9 (2012). http://dx.doi.org/10.1155/2012/759205

[17] G. M. Fioramonti-Calixto, J. Bernegossi, L. Marise de Freitas, C. R. Fontana, M. Chorilli, "Nanotechnology-Based Drug Delivery Systems for Photodynamic Therapy of Cancer: A Review", Molecules 21, 342 (2016).

http://dx.doi.org/10.3390/molecules21030342

\section{Introducción}

A pesar de las ventajas que presenta la Terapia Fotodinámica (TFD) para el tratamiento del cáncer de piel frente a otras técnicas terapéuticas, existen ciertas dificultades que limitan su aplicación para tratar determinados tipos de patologías [1]. Entre dichas dificultades, se encuentra la posibilidad de tratar únicamente lesiones cutáneas superficiales ya que la mayoría de los fotosensibilizadores comercializados actualmente absorben la radiación óptica en la región espectral visible por debajo de $700 \mathrm{~nm}$, donde la penetración de la luz en la piel se limita a unos pocos milímetros. Otro aspecto que puede limitar la respuesta al tratamiento, es una acumulación insuficiente del fotosensibilizador en el tejido tumoral, en 
gran parte debido a la influencia que tiene la capa córnea de la epidermis en el proceso de difusión del precursor hacia las capas más profundas de la piel, [2-3]. Igualmente el carácter hidrofóbico de la mayoría de los fotosensibilizadores que se agregan fácilmente en un medio fisiológico, dificulta la formulación farmacéutica para su administración. De este modo el uso actual de la TFD para tratar ciertos tipos de patologías cutáneas presenta varias limitaciones vinculadas en términos generales a los componentes que intervienen en las reacciones fotodinámicas, como son las relacionadas con el proceso de distribución del fotosensibilizador en el tejido diana, la radiación óptica o las reacciones fotoquímicas que tienen lugar durante el proceso [4].

La Bio-nanotecnología podría ayudar a superar en un futuro gran parte de las limitaciones actuales de la TFD mediante la administración de nanopartículas (NPs) con diferente funcionalidad dentro del contexto del proceso fotodinámico. En términos generales pueden actuar como transductores de energía, ser capaces de generar agentes citotóxicos por sí mismas o bien servir como portadoras del fotosensibilizador [5]. En este último caso, están pensadas para jugar un papel pasivo dentro del contexto fotodinámico y únicamente transportar las moléculas de fotosensibilizador, proporcionando una vía efectiva para superar la naturaleza hidrofóbica de la mayoría de los fotosensibilizadores y lograr una mayor acumulación en el tejido tumoral $[1,5]$. Lo que contribuiría en definitiva a mitigar respuestas al tratamiento incompletas e incluso a evitar daños en el tejido sano.

Entre los diferentes materiales investigados, las NPs de oro son particularmente interesantes por varias razones, entre ellas su facilidad de síntesis, biocompatibilidad y la posibilidad de variar sus propiedades ópticas modificando su forma y tamaño. Se ha demostrado que los conjugados con NPs de oro son excelentes vehículos de entrega de fotosensibilizadores hidrofóbicos ligados a su superficie en cultivos de células tumorales [6-7]. Sin embargo la mayoría de estos estudios están centrados en la síntesis de NPs de oro para mejorar la entrega del fotosensibilizador in vitro por lo que factores dosimétricos con consecuencias directas en la respuesta al tratamiento como son su distribución o su efecto sobre la propagación de la radiación óptica a nivel tisular no se tienen en cuenta. En un tejido biológico, la administración de NPs de oro que actúan con portadoras para los fotosensibilizadores convencionales podría afectar a la absorción óptica y como consecuencia directa a la actualmente limitada profundidad de penetración de la luz en tejido maligno. Por lo que la profundidad de penetración de la luz y en consecuencia la profundidad de tratamiento efectiva podrían verse aún más limitadas a pesar de las mejoras en la entrega del fotosensibilizador. Dejando en todo caso de actuar como un mero elemento pasivo dentro del contexto fotodinámico.

En este trabajo se estudia el efecto de las NPs de oro sobre la distribución de la radiación óptica con fines dosimétricos, cuando actúan como portadoras de diferentes tipos de fotosensibilizador. El análisis predictivo de la posible variación de la profundidad de tratamiento fotodinámico cuando se emplean fotosensibilizadores basados en nanopartículas no ha sido estudiado en profundidad. Entre los fotosensibilizadores, se estudian los de la familia de las porfirinas, ampliamente empleados en dermatooncología y otros que presentan un uso potencial. Para ello se emplea un modelo predictivo que permite analizar los cambios inducidos por la administración de NPs esféricas portadoras del fotosensibilizador sobre la distribución de la radiación óptica en el tejido maligno. A partir de la distribución óptica se estima la profundidad del tratamiento fotodinámico en un carcinoma basocelular nodular considerando diferentes tipos de fotosensibilizadores cuando se entregan tanto de forma convencional como mediante diferentes NPs de oro. Lo que permite analizar su efecto sobre el resultado del tratamiento de la TFD convencional con dichos fotosensibilizadores. En la sección 2 se presenta los fundamentos del modelo empleado para obtener la profundidad del tratamiento fotodinámico cuando se emplean NPs de oro portadoras de fotosensibilizador. La aplicación del modelo sobre un carcinoma basocelular nodular considerando diferentes tipos de NPs y concentraciones, así como diferentes tipos de fotosensibilizadores, se presenta en la sección 3 junto a los resultados obtenidos. Finalmente en la sección 4 se recogen las principales conclusiones del trabajo.

\section{Fundamentos del modelo}

En medios turbios, como es el caso de la mayoría de los tejidos biológicos, cuando el esparcimiento (scattering) es dominante, la teoría del transporte de la radiación permite describir el transporte de energía a través del medio mediante la ecuación del transporte de la radiación [8]. En el estado estacionario, cuando la fuente de luz ha iluminado la muestra de tejido durante el tiempo suficiente de 
forma que la radiación óptica ha alcanzado el equilibrio, y en una región libre de fuentes, la ecuación del transporte de la radiación puede expresarse como en (1). Donde la luz se propaga desde la dirección $s^{\prime}$ a la dirección $s, I(r, \hat{s})$ es la intensidad específica en $\mathrm{W} / \mathrm{m}^{2} \mathrm{sr}, \mu_{a}$ es el coeficiente de absorción, $\mu_{s}$ es el coeficiente de scattering, $p\left(\hat{s}, \hat{s}^{\prime}\right)$ es la función de fase de scattering que contiene las probabilidades de que la luz sea esparcida en diferentes direcciones y $\Omega$ es el ángulo sólido.

$$
\hat{s} \cdot \nabla I(r, \hat{s})=-\left(\mu_{a}+\mu_{s}\right) I(r, \hat{s})+\frac{\mu_{s}}{4 \pi} \int_{4 \pi} p\left(\hat{s} \cdot \hat{s}^{\prime}\right) I\left(r, \hat{s}^{\prime}\right) d \Omega^{\prime}
$$

En el caso particular de los tejidos biológicos la resolución de la ecuación RTT (Radiation Transport Theory) se suele realizar mediante el método de Monte Carlo, ya que proporciona una buena aproximación para resolver problemas relacionados con el transporte de la luz en los tejidos. Entre las diferentes aproximaciones de este método se utiliza la aproximación multicapa de Wang y Jacques [9], que permite definir diferentes capas de tejidos con sus bordes perpendiculares al haz óptico. Esto es de gran utilidad en tejidos estratificados, como es el caso de la piel. En este caso es preciso conocer los parámetros ópticos de los diferentes tejidos así como su grosor. Sin embargo, cuando la luz se propaga a través de un medio biológico que alberga NPs, no sólo es absorbida y esparcida por el tejido, sino también por las propias NPs [10]. Por este motivo, el cálculo de la distribución óptica en el tejido tratado, debe tener en cuenta las propiedades ópticas de las NPs.

El esparcimiento de la luz debido a estructuras esféricas como las NPs consideradas en este trabajo, se puede calcular mediante la teoría de Mie teniendo en cuenta su radio $r$ y el índice de refracción del oro $[11,12]$. Así se pueden calcular las propiedades de scattering y absorción de las NPs de oro como se expresa en (2) a partir de los factores de eficiencia $Q_{i}$ calculados mediante la teoría de Mie. Representando el subíndice $i$ la absorción o el scattering y $N$ la concentración de NPs.

$$
\mu_{i}=\pi r^{2} Q_{i} N
$$

Los factores de eficiencia de extinción, absorción y scattering se pueden calcular mediante las siguientes expresiones:

$$
\begin{gathered}
Q_{e x t}=\frac{2}{x^{2}} \sum_{n=1}^{\infty}(2 n+1) \operatorname{Re}\left(a_{n}+b_{n}\right) \\
Q_{s c a}=\frac{2}{x^{2}} \sum_{n=1}^{\infty}(2 n+1)\left(\left|a_{n}\right|^{2}+\left|b_{n}\right|^{2}\right) \\
Q_{a b s}=Q_{e x t}+Q_{a b s}
\end{gathered}
$$

En estas expresiones, los coeficientes de amplitud $a_{n}$ y $b_{n}$ se calculan mediante las ecuaciones 6 y 7 , en función de las funciones de Bessel esféricas [11]. El índice de refracción de la esfera relativo al del medio circundante es $m, x=k r$ es el parámetro de tamaño, $k=2 \pi / \lambda$ es el número de onda, $\lambda$ es la longitud de onda en el medio circundante, y $\mu_{1}$ es la relación entre la permeabilidad magnética de la esfera y la del medio circundante.

$$
\begin{gathered}
a_{n}=\frac{m^{2} j_{n}(m x)\left[x j_{n}(x)\right]^{\prime}-\mu_{1} j_{n}(x)\left[m x j_{n}(m x)\right]^{\prime}}{m^{2} j_{n}(m x)\left[x h_{n}^{(1)}(x)\right]^{\prime}-\mu_{1} h_{n}^{(1)}(x)\left[m x j_{n}(m x)\right]^{\prime}} \\
b_{n}=\frac{\mu_{1} j_{n}(m x)\left[x j_{n}(x)\right]^{\prime}-j_{n}(x)\left[m x j_{n}(m x)\right]^{\prime}}{\mu_{1} j_{n}(m x)\left[x h_{n}^{(1)}(x)\right]^{\prime}-h_{n}^{(1)}(x)\left[m x j_{n}(m x)\right]^{\prime}}
\end{gathered}
$$

\section{Aplicación del modelo y resultados}

Las propiedades ópticas de las NPs de oro fueron calculadas mediante la teoría de Mie a la longitud de onda adecuada para excitar diferentes tipos de fotosensibilizadores. Un fotosensibilizador de la familia de 
las cianinas cuyas bandas de absorción útiles para TFD caen aproximadamente en el rango espectral comprendido entre 535 y $574 \mathrm{~nm}$, de la familia de las porfirinas cuya banda de absorción espectral está entre 630 y $650 \mathrm{~nm}$, de la familia de las clorinas cuya absorción se encuentra entre 650 y $700 \mathrm{~nm}$ y de la familia de las bacterioclorinas entre 700 y 740/800 nm [5]. Se consideraron NPs esféricas de radios $40 \mathrm{~nm}$ y $80 \mathrm{~nm}$, así como dos concentraciones $\left(2 \cdot 10^{9}\right.$ y $\left.4 \cdot 10^{9} \mathrm{~cm}^{-3}\right)$ distribuidas homogéneamente en un carcinoma basocelular nodular de $6 \mathrm{~mm}$ de profundidad. Las propiedades ópticas de este tipo de tejido tumoral fueron obtenidas a la longitud de onda de interés (coincidente con el valor central de los rangos de absorción máxima anteriormente referidos) para excitar las diferentes moléculas de fotosensibilizador y desencadenar las reacciones fotoquímicas a partir de las cuales se produce el agente citotóxico encargado de la destrucción del tejido durante la TFD [13].

La distribución de la radiación óptica en el carcinoma sin emplear NPs y con diferentes NPs para entregar los diferentes tipos de fotosensibilizador, se obtuvieron mediante el método de Monte Carlo, teniendo en cuenta las propiedades ópticas adecuadas para excitar este tipo de fotosensibilizadores. Se consideró un haz óptico cilíndrico de $0.25 \mathrm{~cm}$ de radio perpendicular al carcinoma y una irradiancia de $200 \mathrm{~mW} / \mathrm{cm}^{2}$. Este haz incide perpendicularmente sobre la capa de $6 \mathrm{~mm}$ de carcinoma basocelular nodular. La distribución óptica en el interior del tejido se obtiene mediante el método de Monte Carlo anteriormente referido. En la Figura 1 se representa la absorción óptica en el tejido cuando se expone a la TFD con los diferentes fotosensibilizadores entregados de forma convencional (Fig. 1 a, c, e y g para la familia de las cianinas, porfirinas, clorinas y bacterioclorinas respectivamente) y empleando NPs de oro (80 $\mathrm{nm}, 4 \cdot 10^{9}$ $\mathrm{cm}^{-3}$ ) como medio de entrega (Fig. $1 \mathrm{~b}, \mathrm{~d}, \mathrm{f}$ y h para la familia de las cianinas, porfirinas, clorinas $\mathrm{y}$ bacterioclorinas respectivamente). Tal y como se puede observar, la presencia de NPs de oro embebidas en el tejido provoca un incremento de la absorción en las regiones más superficiales del tumor y por lo tanto una reducción de la profundidad de penetración óptica en el tejido. Así, la absorción máxima superficial en el caso de las cianinas es del orden de $1.6 \mathrm{~W} / \mathrm{cm}^{3}$ cuando no se emplean nanopartículas, y de casi $2 \mathrm{~W} / \mathrm{cm}^{3}$ cuando se utilizan nanopartículas portadoras. En todos los casos estudiados la entrega de moléculas de fotosensibilizador mediante NPs de oro produjo este mismo efecto, aunque el grado de reducción de la profundidad de penetración óptica depende tanto del tamaño de la NPs como de su concentración en el tumor, llegando a ser significativo en algunos casos.

A partir de la profundidad efectiva de penetración de la luz en el tejido tumoral, dada por $\delta=1 / \mu_{\text {eff }}$ donde $\mu_{\text {eff }}=\sqrt{3 \mu_{a} \mu_{s}^{\prime}}$, cuando se aplica radiación óptica en el rango espectral adecuado para excitar cada fotosensibilizador, tanto administrado convencionalmente como mediante NPs, es posible obtener la profundidad del tratamiento fotodinámico dTFD. Esta última tiene un valor estimado de $d_{T F D} \sim 3-5 \delta$ para la mayoría de los fotosensibilizadores empleados en la práctica clínica [14]. Aunque otras fuentes imponen un límite más restrictivo para que se produzca el efecto biológico de la TFD (necrosis, apoptosis, colapso vascular) y sugieren que ocurre hasta a aproximadamente el doble de la profundidad de penetración de la luz [8]. Empleando este último criterio, se obtuvo la profundidad del tratamiento fotodinámico. La inclusión de NPs de oro como medio de entrega para las moléculas de fotosensibilizador produce una disminución de la profundidad efectiva del tratamiento fotodinámico como consecuencia de una mayor absorción óptica que reduce la penetración de la radiación óptica en el carcinoma. Por lo que la efectividad de la TFD con este tipo de NPs debe ser analizada teniendo en cuenta que pueden empeorar la actualmente limitada penetración de la luz en el medio biológico empleando fotosensibilizadores convencionales. En la Figura 2 se recoge dicho empeoramiento, expresado como reducción de la profundidad del efecto destructivo fotodinámico cuando se emplea un fotosensibilizador sin nanopartículas, considerando diferentes tamaños de NP y diferentes concentraciones.

Atendiendo a los resultados obtenidos la administración de $2 \cdot 10^{9} \mathrm{~cm}^{-3}$ NPs $40 \mathrm{~nm}$ portadoras de un fotosensibilizador de la familia de las clorinas reduce entre un 0.63 y un $3.07 \%$ la profundidad del tratamiento fotodinámico respecto a su administración convencional, siendo el caso de mayor empeoramiento si se compara con otros tipos de fotosensibilizadores (porfirinas 0.54-0.63\%, clorinas 0.26-0.54 \%, bacterioclorinas 0.26-0.34 \%). Al aumentar el tamaño de la nanopartícula a $80 \mathrm{~nm}$ manteniendo la misma concentración, la profundidad de tratamiento se ve reducida aún más en todos los casos, siendo el empeoramiento de entre 7.29 y $9.45 \%$ para las cianinas, $9.37 \%$ para las porfirinas, entre 5.89 y 9.37 \% para las clorinas y de entre 4.54 y 5.89 \% para las bacterioclorinas. La disminución de la profundidad de tratamiento es notablemente mayor cuando se aumenta además la dosis de NPs, llegando a ser de entre 17.04 y $18.6 \%$, entre 17.04 y $17.13 \%$, entre 9.53 y $17.13 \%$ y entre 8.68 y $9.6 \%$ cuando el fotosensibilizador es de la familia de las cianinas, porfirinas, clorinas y bacterioclorinas respectivamente. 
a)

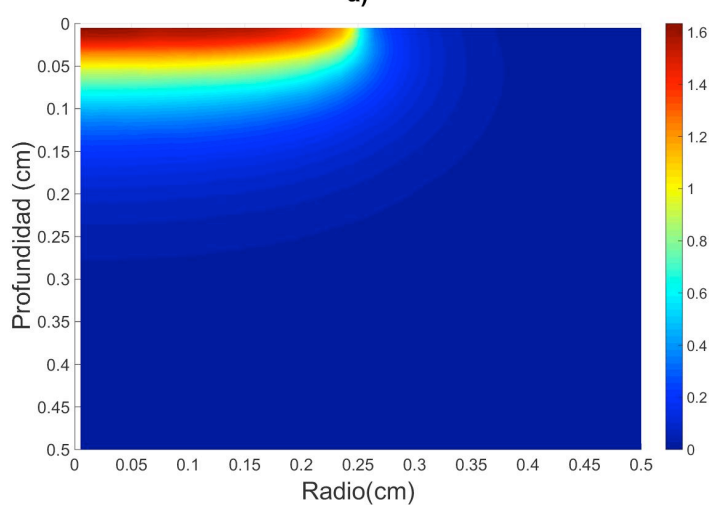

c)

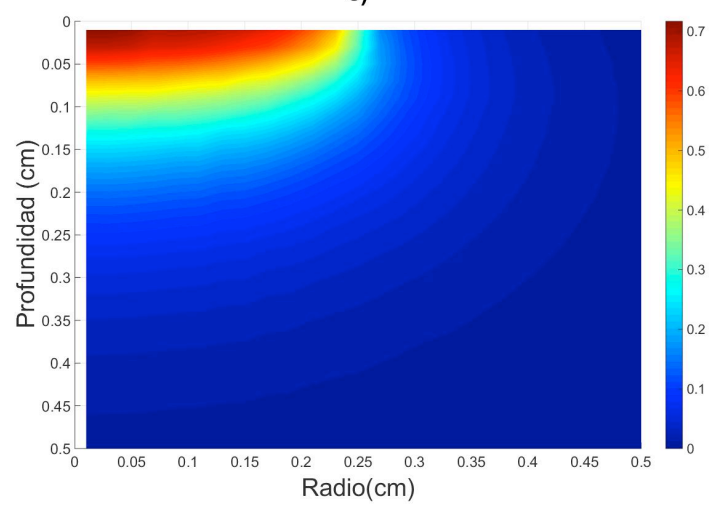

e)

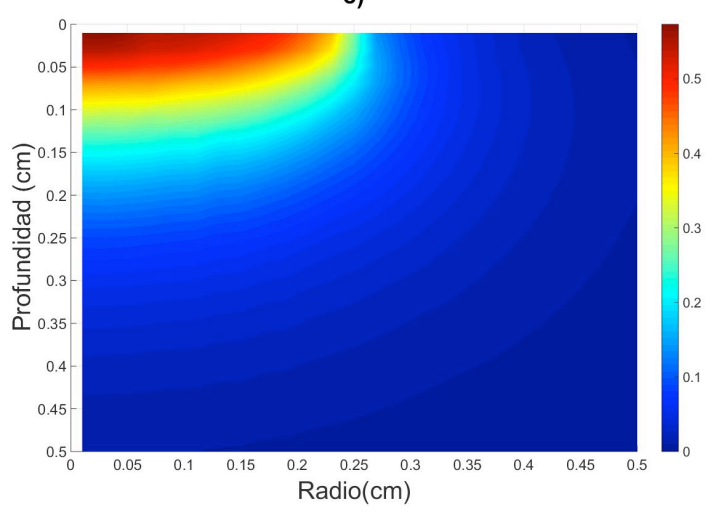

g)

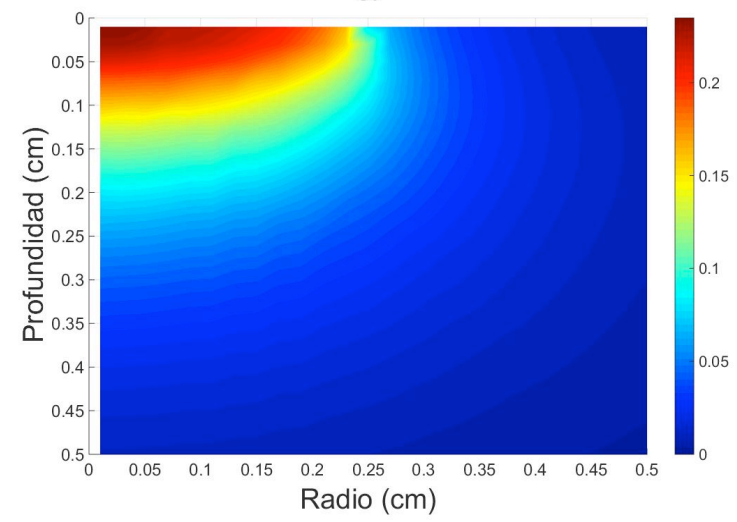

b)

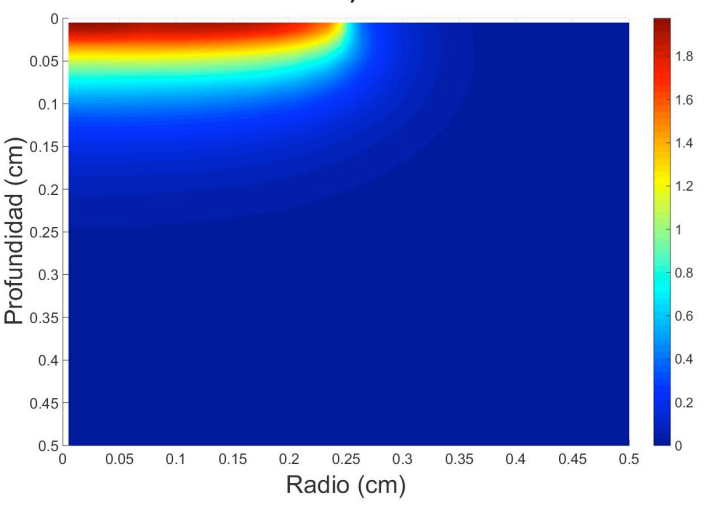

d)

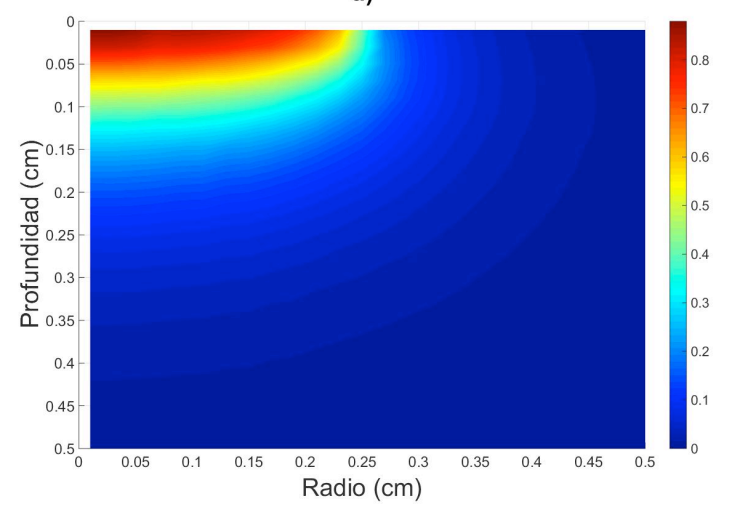

f)

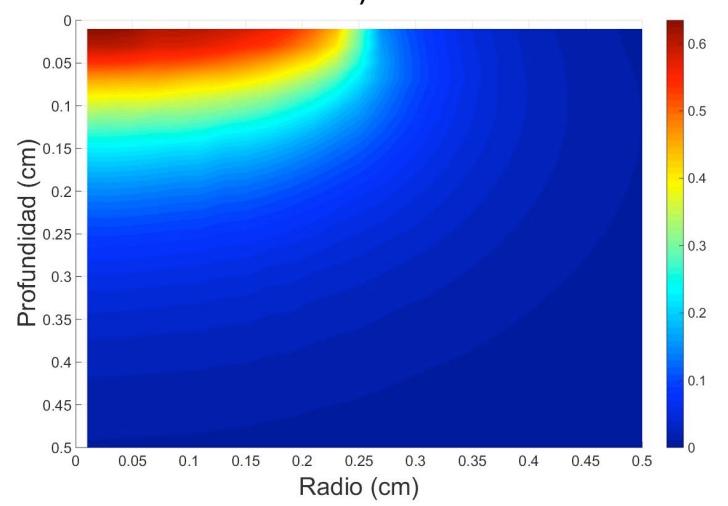

h)

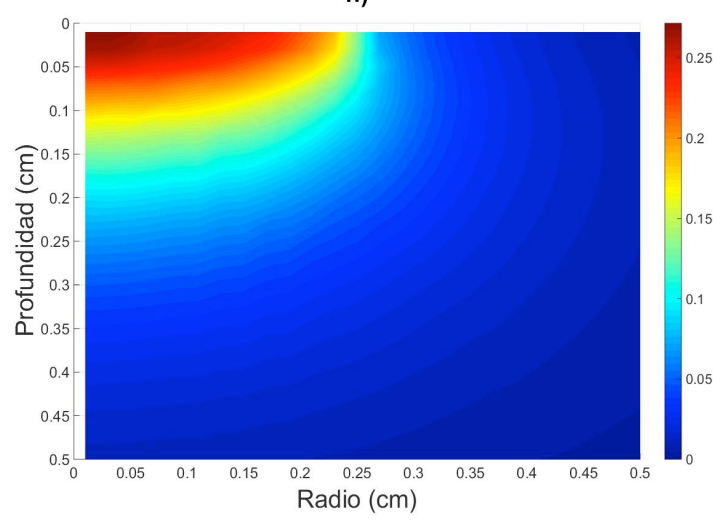

Fig.1. Absorción óptica $\left(\mathrm{W} / \mathrm{cm}^{3}\right.$ ) en el tumor cuando se somete a TFD empleando un fotosensibilizador convencional de la familia de las a) cianinas, c) porfirinas, e) clorinas y g) bacterioclorinas y cuando dichos fotosensibilizadores (b) cianinas, d) porfirinas, f) clorinas $\mathrm{y} \mathrm{h}$ ) bacterioclorinas) se entregan mediante NPs de oro $\left(80 \mathrm{~nm}, 4 \cdot 10^{9} \mathrm{~cm}^{-3}\right)$. 

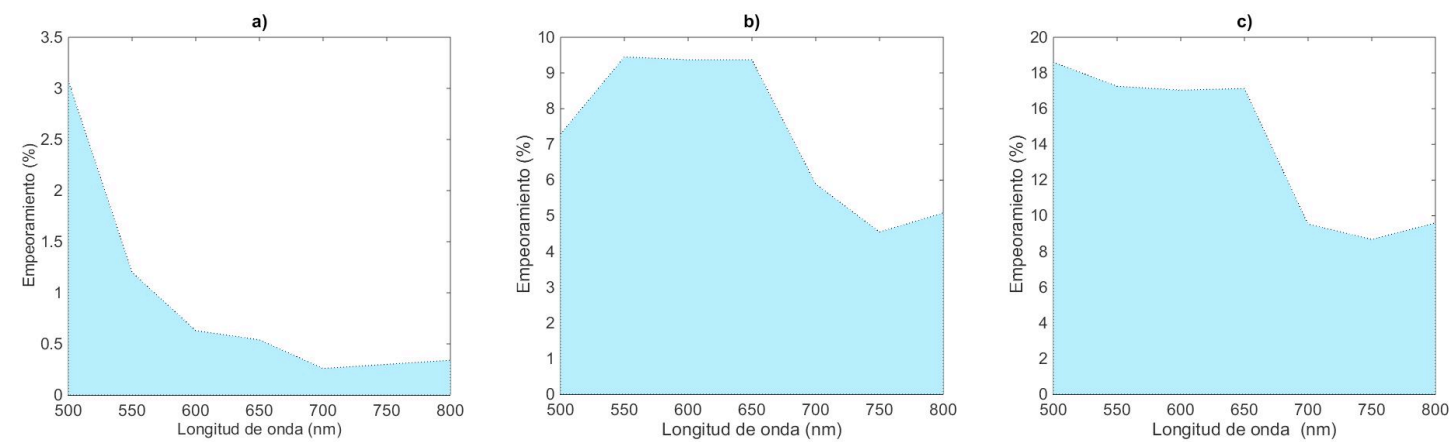

Fig.2. Empeoramiento (\%) de la profundidad del tratamiento fotodinámico respecto al uso del fotosensibilizador convencional cuando éste se entrega mediante a) una concentración de $2 \cdot 10^{9} \mathrm{~cm}^{-3} \mathrm{NPs}$ de $40 \mathrm{~nm}$, b) la misma concentración pero con NPs del doble de radio y c) el doble de concentración $\left(4 \cdot 10^{9} \mathrm{~cm}^{-3}\right)$.

Los resultados mostrados en la gráfica izquierda de la Figura 3 muestran en términos porcentuales cuál de los dos parámetros dosimétricos relacionados con la administración de nanopartículas (tamaño o dosis) produce una disminución más significativa de la profundidad terapéutica del tratamiento. Como se puede observar, duplicar la concentración de nanopartículas implica un empeoramiento más significativo que duplicar el radio de las NPs o viceversa dependiendo del rango de excitación espectral empleado para la fotoactivación de las moléculas de un determinado fotosensibilizador. En términos generales se puede observar un primer rango espectral entre 500 y aproximadamente $550 \mathrm{~nm}$, coincidente con el rango de excitación de los fotosensibilizadores de la familia de las cianinas (535-574 $\mathrm{nm}$ ), en el que duplicar la concentración de nanopartículas provoca un empeoramiento más significativo en la profundidad del tratamiento que duplicar el radio de las nanopartículas puesto que el aumento de este último desplaza la banda de resonancia plasmónica localizada en torno a $500 \mathrm{~nm}$ para las nanopartículas de menor radio hacia longitudes de onda del orden de $570 \mathrm{~nm}$. En el segundo tramo (entre aproximadamente $550 \mathrm{~nm}$ y $800 \mathrm{~nm}$ ) el empeoramiento es mayor si se dobla el radio de la nanopartícula en lugar de aumentar al doble la dosis de nanopartículas. Tal y como se puede observar, tanto duplicar la concentración como duplicar el radio de las nanopartículas portadoras de cianinas excitadas a aproximadamente $550 \mathrm{~nm}$ produce un empeoramiento similar al igual que en el rango espectral entre 750 y $800 \mathrm{~nm}$ correspondiente a las bandas de excitación de las bacterioclorinas. La diferencia absoluta en puntos porcentuales entre los dos ajustes dosimétricos estudiados (modificar el radio de la nanopartícula y la concentración) se recoge en la gráfica derecha de la Figura 3, donde se pone de manifiesto la importancia de su adecuada elección atendiendo a la longitud de onda de excitación empleada para excitar las moléculas de un determinado fotosensibilizador. Como se puede observar, el efecto de duplicar el radio de la nanopartícula o aumentar al doble la concentración, produce diferencias menos significativas (entre un 0.1 y un $2 \%$ entre 550 y 800 $\mathrm{nm}$ ) en términos de empeoramiento de la profundidad que para el rango de longitudes de onda más bajas, donde la diferencia puede llegar hasta el $7 \%$.
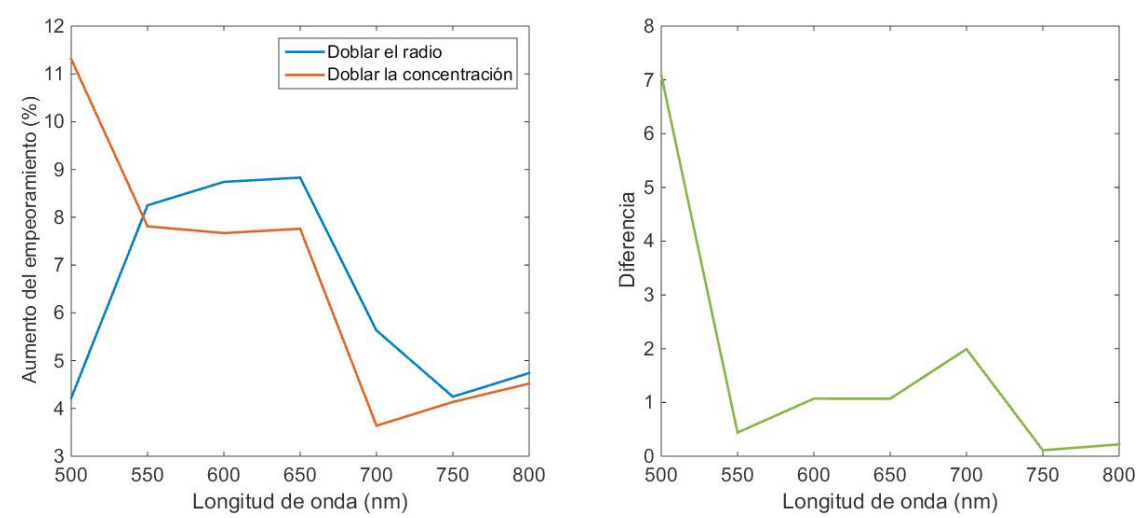

Fig.3. Empeoramiento sobre la profundidad del tratamiento que supone duplicar el radio de las NPs y duplicar su concentración (gráfica izquierda) y diferencia absoluta entre ambos (gráfica derecha). 
Los resultados obtenidos ponen de manifiesto la importancia del tamaño de las NPs administradas, así como de su concentración en el medio biológico. De modo que tanto un aumento del radio como de la dosis administrada supone una disminución de la profundidad de los efectos del tratamiento. Siendo esta última especialmente crítica cuando se trata de eliminar tumores que se extienden hasta una determinada profundidad. Por lo que el uso de modelos predictivos [15] como el empleado en este trabajo podría ser de gran utilidad para descartar la administración de este tipo de NPs cuando se planifica el tratamiento mediante TFD sobre carcinomas con una determinada estructura morfológica [16, 17]. Igualmente, se evidencia la necesidad de seleccionar su tamaño atendiendo al tipo de fotosensibilizador empleado. Ya que si su rango de excitación espectral coincide con la banda de resonancia plasmónica de las NPs empleadas como medio de entrega, dicha reducción será aún más significativa. Siendo todos estos, aspectos a tener en cuenta para evitar una posible persistencia tumoral tras el tratamiento fotodinámico. A la vista de los resultados obtenidos cabría la posibilidad de cuestionarse el papel pasivo que se atribuye a las NPs portadoras de fotosensibilizador en la TFD.

\section{Conclusiones}

El uso combinado de NPs y TFD en dermatooncología podría ayudar a superar en un futuro gran parte de las limitaciones actuales de esta técnica. En este contexto, las NPs pueden tener diferentes funciones, entre las cuales destaca su uso como portadora del fotosensibilizador para superar la naturaleza hidrofóbica de la mayoría de los fotosensibilizadores y lograr una mayor acumulación en el tejido tumoral. En este caso su función se limita únicamente al transporte del fotosensibilizador por lo que se les atribuye un papel pasivo dentro del proceso fotodinámico. Entre los diferentes materiales estudiados, se ha demostrado que los conjugados con NPs de oro son excelentes vehículos de entrega de fotosensibilizadores hidrofóbicos in vitro. Sin embargo, su estudio a nivel celular no considera factores dosimétricos con consecuencias directas en la respuesta al tratamiento como la distribución de la dosis administrada o su efecto sobre la propagación de la radiación óptica a nivel tisular. Este último con consecuencias directas sobre la actualmente limitada profundidad de penetración de la luz en tejido maligno y en consecuencia sobre la profundidad de tratamiento efectiva. Por lo que a pesar de las mejoras en la entrega del fotosensibilizador, este tipo de NPs podría dejar de actuar como un mero elemento pasivo dentro del contexto fotodinámico llegando incluso a limitar los efectos del tratamiento dependiendo de múltiples factores tan diversos como el tamaño de la NP y la dosis administrada, el tipo de tejido y su morfología o el tipo de fotosensibilizador empleado entre otros. Por lo que una planificación adecuada del tratamiento es indispensable para evitar una posible persistencia tumoral. Para tal fin el uso de modelos predictivos como el empleado en este trabajo presenta un gran interés. Dicho modelo se ha empleado para estudiar el efecto de las NPs de oro sobre la distribución de la radiación óptica con fines dosimétricos, cuando actúan como portadoras de diferentes tipos de fotosensibilizador. Entre ellos, fotosensibilizadores de la familia de las porfirinas ampliamente empleados en dermatooncología y otros que presentan un uso potencial. Dicho modelo ha permitido analizar los cambios inducidos por la administración de NPs esféricas portadoras de diferentes fotosensibilizadores sobre la distribución de la radiación óptica en un carcinoma basocelular nodular. A partir de la distribución óptica se estimó la profundidad del tratamiento fotodinámico y se comparó con la entrega convencional del fotosensibilizador. Los resultados obtenidos destacan la importancia de elegir adecuadamente tanto el tamaño como la dosis de NPs administradas atendiendo al tipo de fotosensibilizador durante la planificación del tratamiento para no limitar la profundidad de los efectos terapéuticos, especialmente en carcinomas de una cierta profundidad.

\section{Agradecimientos}

Este trabajo ha sido parcialmente financiado por el proyecto del Plan Nacional de I+D+i "Estudio de las propiedades ópticas y magnéticas del ZnO impurificado con metales de transición y sus aplicaciones en biomedicina" (MAT2012-38664-C02-01), cofinanciado con fondos FEDER, y por la Fundación San Cándido. 